\title{
Vulnerabilidad al virus de la hepatitis B y prácticas preventivas en trabajadores de un hospital de Guatemala
}

\author{
Hepatitis B virus vulnerability and preventive practices among \\ workers at a Guatemalan Hospital
}

\author{
Dorian Ramírez-Flores $^{1 *}$, Patricia Vélez-Möller ${ }^{2,3}$, Magda Velásquez-Tohom ${ }^{1}$ \\ ${ }^{1}$ Centro de Investigaciones de las Ciencias de la Salud (CICS) y ${ }^{2}$ Escuela de Estudios de Postgrado, \\ Facultad de Ciencias Médicas, Universidad de San Carlos de Guatemala (Usac), Guatemala \\ ${ }^{3}$ Asociación Guatemalteca del Hígado, Guatemala
}

*Autor al que se dirige la correspondencia: dorianramirez@gmail.com

Recibido: 23 de enero 2017 / Revisión: 04 de abril 2017 / Aceptado: 23 de junio 2017

\section{Resumen}

a hepatitis B (HB) es un problema de relevancia mundial para la salud pública y ocupacional. Diferentes
informes indican una seroprevalencia intermedia del altamente transmisible virus de la hepatitis B (VHB)
en Guatemala, que impone un riesgo sobre los trabajadores de salud (TS). Este artículo describe la adherencia a
prácticas preventivas, la seroprevalencia de anticuerpos contra el antígeno de superficie (anti-HBs) y el riesgo de
accidente ocupacional punzocortante en TS del Hospital Nacional Pedro de Bethancourt de Guatemala. En $121 \mathrm{TS}$
voluntarios, se estimó la prevalencia de exposiciones, prácticas preventivas, concentración de anticuerpos anti-HBs,
utilizando Elisa, así como odds ratios (OR) de diferentes factores de riesgo para heridas punzocortantes. Tener
contacto con pacientes ( $69 \%$ ) y manipular material punzocortante (63\%) o biológico (54\%) fueron identificados
como peligros de exposición al VHB. Los TS entrevistados indicaron respetar las normas de bioseguridad (75\%),
utilizar guantes (69\%) y estar vacunados ( $48 \%$ ). La prevalencia de anti-HBs fue del $43 \%$. El riesgo de accidentes
punzocortantes fue 9 veces mayor para médicos (OR, 8.9 ; IC $95 \%$ [1.7, 63$] ; p=.0017)$ y 3 veces mayor para
enfermeros (OR, 3.2 ; IC $95 \%$ [1.15, 9.1$] ; p=.0121)$, que para el resto del personal. Las prácticas de bioseguridad
e inmunización son deficientes entre los TS del hospital, a pesar de ser una población con riesgo de infección por
el VHB. Se recomienda fortalecer las competencias sobre bioseguridad y el cumplimiento de medidas preventivas
en el hospital, así como institucionalizar la vacunación contra VHB para todos los TS.

Palabras claves: Salud laboral, infección hospitalaria, control de enfermedades transmisibles, salud pública

\section{Abstract}

$\mathrm{H}$ epatitis B is a major, global issue for both public and occupational health. Reports indicate an intermediate seroprevalence of the highly transmissible hepatitis B virus (HBV) in Guatemala, imposing a high risk for healthcare workers (HCWs). We describe the adherence to preventive practices, seroprevalence of surface antigen's antibodies (anti-HBs) and the risk of puncture-related occupational accidents with medical sharps among HCWs, at Guatemalan Pedro de Bethancourt National Hospital (PBNH). Prevalence of exposure and prevention practices was estimated in 121 volunteering HCWs, as well as serum concentrations of anti-HBs, using ELISA. Odds ratios $(\mathrm{OR})$ of risk factors for puncture wounds with medical sharps were estimated. Contact with patients $(69 \%)$, medical sharps (63\%) and biological material (54\%) were identified as a risk of exposure to HBV. Of all volunteers interviewed, $75 \%$ reported compliance with biosafety standards, $69 \%$ use surgical gloves and $48 \%$ reported having been vaccinated. Seroprevalence of anti-HBs was $43 \%$. The risk of injuries with medical sharps was 9 -fold among physicians and 3-fold among nurses, as compared with the rest of the hospital staff (OR, 8.9, 95\% CI [1.7, 63], $p$ $=.0017$; OR, 3.2, 95\% CI) $[1.15,9.1], p=.0121$, respectively). Biosecurity and immunization practices are poor among hospital HCWs, despite being a population at high risk for HBV infection. We recommend that biosecurity capacity building and compliance with preventive practices be ensured. A sustainable HBV vaccination program for all HCWs should be institutionalized. 


\section{Introducción}

La hepatitis B (HB) es un problema mundial de salud pública. Se estiman 2,000 millones de personas infectadas con el virus de la hepatitis B (VHB) en el mundo (McMahon, 2005), 240 millones con hepatitis crónica y 686 mil muertes anuales por complicaciones de la enfermedad (World Health Organization [WHO], 2016b). La infección crónica por el VHB se asocia a muerte por cirrosis hepática y carcinoma hepatocelular (Franciscus, Highleyman, \& Kukka, 2008; Naghavi et al., 2015). Después del tabaco, el VHB es el segundo carcinógeno conocido, y causa el 50\% de los casos de cáncer hepático (Ben-Ari, Weitzman, \& Safran, 2015; El-Serag, 2011).

El endemismo del VHB en Latinoamérica no ha sido bien caracterizado. Las prevalencias calculadas por país podrían estar sesgadas, debido a que se han basado en datos parciales, no representativos. En Guatemala, por ejemplo, la seroprevalencia es intermedia $(>2 \%<$ $8 \%$ ) de acuerdo con unos autores (Tanaka, 2000; Trépo, Chan, \& Lok, 2014) y baja $(<2 \%)$, según otros (Ott, Stevens, Groeger, \& Wiersma, 2012; Schweitzer, Horn, Mikolajczyk, Krause, \& Ott, 2015).

Los trabajadores de salud (TS) son particularmente vulnerables por su exposición, directa e indirecta, a sangre y otros fluidos corporales potencialmente infectados y el alto riesgo que tienen de sufrir accidentes ocupacionales que involucran lesiones con material punzocortante contaminado (Cubides \& Suárez, 2009; Mendoza \& Samalvides, 2005). Velásquez y Partanen (2007) informan que el 64\% de los TS del Hospital Nacional Pedro de Bethancourt (HNPB) tuvo contacto con sangre y únicamente el $48 \%$ se encontraba inmunizado contra el VHB (Velásquez \& Partanen, 2007).

El Plan de Acción Mundial por la Salud de los Trabajadores 2008-2017 de la Organización Mundial de la Salud (OMS) recomienda inmunizar a todo el personal sanitario contra la $\mathrm{HB}$, enfermedad considerada prioritaria para ser eliminada o erradicada (2009). El Programa Nacional de Inmunizaciones (PNI) del Ministerio de Salud Pública y Asistencia Social (MSPAS) emitió en 2011 los lineamientos técnicos para la vacunación contra VHB al personal de salud de Guatemala.

El HNPB ha efectuado jornadas de vacunación contra el VHB en TS en 1999 y 2005, sin embargo, no se tienen registros del número de vacunados ni de las dosis administradas. Con el propósito de evaluar riesgos y planificar intervenciones, han surgido iniciativas locales de promoción y prevención dirigidas a TS, las que no han sido respaldadas por una política nacional de salud y seguridad ocupacional (SSO), por ejemplo, la detección de anticuerpos anti-HBs en el personal que se contrata, la vacunación obligatoria, el acceso a vacunas, entre otras acciones.

La infección de un TS es relevante para la salud pública, tanto por su propia salud como un derecho constitucional, laboral y humano, como por la posibilidad de convertirse en transmisor de la infección (Lewis, Enfield, \& Sifri, 2015), aun cuando se ha descrito poca transmisión de TS a pacientes. El interés por la protección de los TS contra la infección por el VHB ha crecido debido al impacto negativo en la calidad de vida del TS infectado y a su papel como potencial agente de contagio para otras personas (Lewis et al., 2015; Zumaeta et al., 2001).

La caracterización de los grupos con mayor riesgo, las formas de exposición al $\mathrm{VBH}$, los conocimientos y prácticas preventivas, la seroprevalencia de anticuerpos contra el antígeno de superficie del VHB (anti-HBs) y el riesgo de accidente ocupacional punzocortante entre TS del HNPB, aportará información para diseñar acciones y optimizar los recursos para la prevención de la exposición al VHB.

\section{Materiales y métodos}

\section{Diseño y población de estudio}

En este estudio auxiliar (Randy \& Hearst, 2008) se incluyeron 121 de 587 TS del HNPB, quienes participaban en un estudio sobre riesgo metabólico entre junio y julio de 2010. Se les informó del objetivo del estudio y se les solicitó autorización para utilizar una parte de la muestra de sangre extraída en el estudio primario.

\section{Recolección de datos}

Se obtuvo datos demográficos, antecedentes de riesgo, exposición ocupacional a sangre y otros fluidos corporales, prácticas de prevención y protección ocupacional. El cuestionario fue administrado por profesores de la Facultad de Ciencias Médicas de la Usac, quienes, al momento del estudio, no trabajaban en el HNPB ni tenían una posición jerárquica que hubiera inducido respuestas por complacencia de parte de los participantes.

De la misma muestra de sangre extraída para el estudio primario, siguiendo las normas de bioseguridad, se separó $1 \mathrm{cc}$ de suero que fue vertido en viales de 0.5 
cc. Las muestras fueron transportadas hacia el Laboratorio Multidisciplinario de la Facultad de Ciencias Médicas de la Usac y almacenadas en refrigeración, hasta su procesamiento. Se determinó la presencia de anti-HBs utilizando una prueba comercial (Monolisa ${ }^{\circledR}$ Anti-HBs Plus), según las indicaciones del fabricante y las normas de buenas prácticas de laboratorio.

El equipo de investigadores fue estandarizado, así como los materiales, instrumentos de medición y el cuestionario. Se adiestró y supervisó al personal técnico de laboratorio para el procesamiento de las muestras.

\section{Análisis estadístico}

Fueron calculados los porcentajes de las variables categóricas, así como la media y la desviación estándar de las variables continuas. Se usó la prueba $t$ de Student para la comparación de dos medias. Se estimó la prevalencia de anti-HBs y los factores de riesgo para accidentes ocupacionales punzocortantes fueron explorados a través del odds ratio (OR) más intervalos de confianza del 95\% (IC 95\%) y la significancia estadística a nivel del $5 \%$. Los cálculos y las pruebas estadísticas fueron realizados con el programa Stata 12.1 (StataCorp. 2011. Stata Statistical Software: Release 12. College Station, TX: StataCorp LP.)

El protocolo del estudio fue evaluado por el Comité de Docencia e Investigación (CDI) y autorizado por la Dirección del HNPB. Todos los participantes dieron su consentimiento informado escrito para separar suero de las muestras de sangre extraídas para el estudio primario y medir anti-HBs, así como para aportar datos demográficos, de accidentes laborales y de exposición al VHB. Durante el reclutamiento, se acordó con cada participante el periodo para entregarle su resultado y darle orientación. Se recomendó vacunación o pruebas adicionales más referencia al Comité de Infecciones Nosocomiales (CIN) del hospital, según cada resultado individual. La directora del laboratorio del HNPB, integrante del CIN, se encargó de entregar los resultados a los participantes que no acudieron durante el periodo convenido, y refirió a los casos positivos sin historia de vacunación al CNI para las recomendaciones pertinentes, incluida la de iniciar o completar la vacunación en el Instituto Guatemalteco de Seguridad Social (IGSS), a los TS afiliados.

\section{Resultados}

La descripción de la edad, sexo y cargo que ocupan los participantes se muestra en la Tabla 1. No se encontró diferencia en la edad media entre hombres y mujeres $(p=.067)$.

Según la profesión de los participantes, la muestra incluyó 23 auxiliares de enfermería (19\%), 11 médicos (9.1\%), 7 técnicos de laboratorio (5.8\%), 6 enfermeras profesionales $(5 \%), 3$ terapistas respiratorios $(2.5 \%)$, 2 licenciadas en enfermería (1.7\%), 2 nutricionistas $(1.7 \%)$ y otros profesionales o técnicos sanitarios 6 (5\%); mientras que personal no sanitario incluyó 15 contadores $(12.4 \%), 10$ secretarias $(8.3 \%)$ y otros, 20 (16.5\%). Sin profesión específica, 16 sujetos (13\%), quienes laboran en los departamentos de mantenimiento, cocina, seguridad y conserjería.

Según el departamento donde laboran, 24 (19.8\%) estaban asignados a farmacia interna e intendencia, 9 (7\%) a nutrición, $24(19.8 \%)$ a consulta externa, enfermería, laboratorio y seguridad, $10(8.3 \%)$ a cirugía y pediatría y 8 a medicina interna y radiología (6.6\%). La afiliación al IGSS fue marcadamente menor en TS

Tabla 1

Características de la población de estudio $(n=121)$

\begin{tabular}{lcc}
\hline Edad & Media & DE \pm \\
\hline Mujeres & 40.38 & 9.9 \\
Hombres & 43.76 & 8.8 \\
Total & 41.55 & 9.7 \\
\hline Cargo que ocupa & Número & $\%$ \\
\hline Administrativo & 26 & 21.5 \\
Personal de enfermería & 24 & 19.8 \\
Seguridad y conserjería & 16 & 13.2 \\
Personal médico & 12 & 9.9 \\
Auxiliar bodega/farmacia & 12 & 9.9 \\
Otro personal de salud ${ }^{\text {a }}$ & 10 & 8.3 \\
Nutrición/cocina & 8 & 6.6 \\
Personal de laboratorio & 7 & 5.8 \\
Mantenimiento/transporte & 6 & 5.0 \\
\hline
\end{tabular}

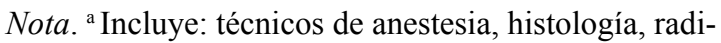
ología, terapia respiratoria y consejeros para pacientes con VIH-sida. 


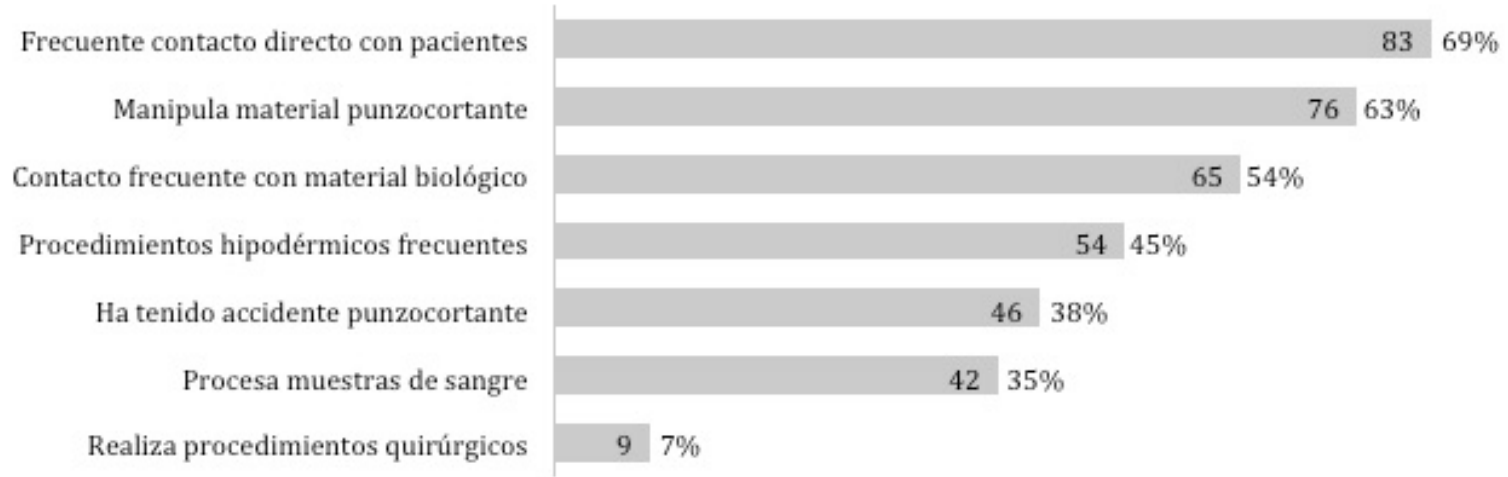

Figura 1. Eventos con riesgo de exposición ocupacional a sangre y otros fluidos corporales.

con menos de 5 años de trabajar en el hospital, con un promedio de edad de 32.5 años $(\mathrm{DE}=8.65)$.

Se determinó como factores de exposición no laboral al VHB cirugía previa 86 (71.1\%), prácticas sexuales sin protección $70(57.85 \%)$ y haber tenido varias parejas sexuales 23 (19\%). Los riesgos de exposición laboral más frecuentes a sangre y fluidos corporales se muestran en la Figura 1.

Indicaron haber tenido accidentes laborales con material punzocortante 46 TS (38\%), 33 mujeres (71.7\%). Únicamente 29 (63\%) notificaron el accidente al CIN del hospital y 25 (54\%) tuvieron seguimiento para VIH, pero no para VHB.

Los factores relacionados significativamente con el riesgo de accidente ocupacional punzocortante se muestran en la Tabla 2.

Las respuestas relacionadas con conocimientos y cumplimiento de las prácticas preventivas se muestran en la Tabla 3. Se encontró a 58 (47.9\%) vacunados contra el VHB, de los que 43 (74\%) recordaron el tiempo de vacunación (rango de 1 a 14 años). La Tabla 4 muestra los porcentajes de TS que realizan procedimientos de riesgo, y los que refirieron conocer y cumplir las normas hospitalarias de bioseguridad.

Se detectaron anticuerpos anti-HBs en 52 participantes (43\%; IC 95\% [34.01, 52.29]). El porcentaje de «Reactivo anti-HBs» es menor en el grupo «No vacunado» y aumenta conforme aumenta el número de dosis, hasta un $79 \%$ en el grupo «Vacunados con tres dosis» (Tabla 5). Este $43 \%$ de seroprevalencia fue similar al $47.9 \%$ de cobertura de vacunación (valor $p$ para la prueba de diferencia entre dos proporciones $=.2806$ ). La Tabla 6 muestra reactividad anti-HBs, según antecedente de vacunación contra hepatitis $\mathrm{B}$ y número de dosis recibidas en un subgrupo de $58 \mathrm{TS}$ que tienen contacto directo con pacientes y alto riesgo de contagio (personal médico, de enfermería, nutrición, laboratorio, terapia respiratoria y radiología), en el que la prevalencia aumentó del $26 \%$ en los no vacunados, al $86 \%$ en los vacunados con tres dosis.

\section{Discusión}

Presentar anticuerpos anti-HBs en suero implica haber tenido contacto previo con el VHB. En el presente estudio se exploraron las posibles asociaciones ocupacionales y no ocupacionales, con el objetivo de reforzar la evidencia del impacto positivo que tiene la vacunación contra el VHB en la salud y seguridad de los TS. La participación por edad, sexo y profesión fue similar a la de otros estudios (Burnett et al., 2011). Similares antecedentes personales y familiares han sido reportados en otros estudios como factores de riesgo para la infección por el VHB (Chacaltana \& Espinoza, 2008; Lok \& McMahon, 2009; Suárez, Mursuli, Pérez, \& Sánchez, 2005; Weinbaum, Mast, \& Ward, 2009), lo que confirma que los TS también tienen riesgo de infección no laboral por el VHB.

La presencia de diversas formas de exposición ocupacional a fluidos corporales a través de diferentes fuentes indica que la población estudiada es susceptible 
Tabla 2

Riesgo de accidente punzocortante, según factor predisponente a exposición ocupacional, en 121 trabajadores hospitalarios

\begin{tabular}{lccc}
\hline Factor predisponente & Frec. & OR (IC 95\%) & $p$ \\
\hline Tener contacto con pacientes $^{*}$ & $41 / 83$ & $8.7[2.84,26.74]$ & $<.001$ \\
Contacto con material biológico $^{\dagger}$ & $37 / 65$ & $6.9[2.90,16.40]$ & $<.001$ \\
Manipular material punzocortante $^{\ddagger}$ & $39 / 76$ & $5.7[2.27,14.40]$ & $<.001$ \\
Cargo de enfermero auxiliar & $14 / 23$ & $3.2[1.15,9.10]$ & .012 \\
Ser médico tratante & $9 / 11$ & $8.9[1.66,62.97]$ & .002 \\
\hline
\end{tabular}

Nota. *Incluye personal médico, de enfermería y otro personal del hospital que entra en contacto frecuente con pacientes y los predispone a accidentes punzocortantes, independientemente de la naturaleza de dicho contacto. ${ }^{\dagger}$ Incluye fluidos corporales, tejidos humanos y desechos biológicos, como compresas, gazas, algodones, etc. ${ }^{\star}$ Incluye agujas, bisturís, tijeras, vidrio, y otros que pueden causar heridas punzocortantes.

Tabla 3

Conocimientos y prácticas de prevención contra la infección por VHB

\begin{tabular}{lcccc}
\hline \multirow{2}{*}{ Conocimientos y prácticas* } & \multicolumn{2}{c}{$\left(n=121^{*}\right)$} & \multicolumn{2}{c}{$\left(n=58^{*}\right)$} \\
\cline { 2 - 5 } Conoce normas de bioseguridad & Sí & $\%$ & Sí & \% \\
Cumple siempre con las normas de bioseguridad & 107 & 88.4 & 57 & 98.3 \\
Recibió del hospital capacitación en bioseguridad & 89 & 73.6 & 48 & 82.8 \\
Realiza procedimientos de riesgo & 96 & 79.3 & 53 & 91.4 \\
Usa guantes durante los procedimientos de riesgo & 76 & 62.8 & 54 & 93.1 \\
Está vacunado contra hepatitis B & 70 & 57.8 & 42 & 73.7 \\
\hline
\end{tabular}

Nota. *Se presenta el número y el porcentaje tanto para el total de 121 participantes, como para el grupo de 58 sujetos que tienen contacto directo con pacientes: personal médico, de enfermería, nutrición, laboratorio, terapia respiratoria y radiología.

Tabla 4

Prácticas preventivas contra VHB de trabajadores que indicaron realizar procedimientos de riesgo

\begin{tabular}{llc}
\hline Prevención y protección $\left(n=76^{*}\right)$ & Sí & $\%$ \\
\hline Conoce las normas de bioseguridad & 73 & 96.1 \\
Cumple siempre con las normas de bioseguridad & 59 & 77.6 \\
Recibió del hospital capacitación en bioseguridad & 67 & 88.2 \\
Está vacunado contra la hepatitis B & 42 & 55.3 \\
\hline
\end{tabular}

Nota. *Incluye 58 sujetos que tienen contacto directo con pacientes y alto riesgo de contagio, como personal médico, de enfermería, nutrición, laboratorio, terapia respiratoria y radiología, más 18 sujetos que manipulan residuos hospitalarios. 
Tabla 5

Prueba anti-HBs reactiva según antecedente de vacunación contra hepatitis $B$ y número de dosis recibidas en la totalidad de la muestra

\begin{tabular}{lcccc}
\hline \multirow{2}{*}{ Vacunación y dosis $n=121$} & \multicolumn{2}{c}{ Vacunación } & \multicolumn{2}{c}{ Reactivo anti-HBs } \\
\cline { 2 - 5 } No vacunado & Frec & $\%$ & Frec & 22.9 \\
Vacunados con 1 dosis & 48 & 39.7 & 11 & 28.6 \\
Vacunados con 2 dosis & 7 & 5.8 & 7 & 58.3 \\
Vacunados con 3 dosis & 12 & 9.9 & 23 & 79.3 \\
Vacunado, pero no sabe número de dosis & 29 & 23.9 & 5 & 50.0 \\
No sabe si está vacunado & 10 & 8.3 & 4 & 26.7 \\
\hline
\end{tabular}

Tabla 6

Prueba anti-HBs reactiva según antecedente de vacunación y número de dosis recibidas, en el grupo de trabajadores con mayor riesgo

\begin{tabular}{lcccc}
\hline \multirow{2}{*}{ Vacunación y dosis $n=58^{*}$} & \multicolumn{2}{c}{ Vacunación } & \multicolumn{2}{c}{ Reactivo anti-HBs } \\
\cline { 2 - 5 } & Frec & $\%$ & Frec & 26.3 \\
\hline No vacunado & 19 & 32.8 & 5 & 33.3 \\
Vacunados con 1 dosis & 3 & 9.7 & 1 & 71.4 \\
Vacunados con 2 dosis & 7 & 22.6 & 5 & 85.7 \\
Vacunados con 3 dosis & 21 & 67.7 & 18 & 33.3 \\
Vacunado, pero no sabe número de dosis & 3 & 5.2 & 1 & 20.0 \\
No sabe si está vacunado & 5 & 8.6 & & 1 \\
\hline
\end{tabular}

Nota. ${ }^{\star}$ Incluye sólo los sujetos que tienen contacto directo con pacientes y alto riesgo de contagio: personal médico, de enfermería, nutrición, laboratorio, terapia respiratoria y radiología.

a contacto ocupacional con el VHB. En estudios similares se ha encontrado que la exposición ocupacional de TS al VHB, a través de sangre y otros fluidos corporales, depende de factores como la prevalencia de la $\mathrm{HB}$ en la población, las prácticas preventivas y el riesgo de incidentes ocupacionales con heridas punzocortantes. Los incidentes de exposición se producen con mayor frecuencia durante procedimientos asistenciales, pero también los sufren TS que pueden entrar en contacto con material punzocortante (Mendoza, Barrientos, Vasquez, \& Panizza, 2001; Morelos, Ramírez, Sánchez, Chavarín, \& Meléndez-Herrada, 2014). El personal de cocina, habitualmente considerado de bajo riesgo ocupacional por no tener contacto directo con los pacientes, informó que, al recoger las bandejas de comida de los pacientes, han encontrado en ellas agujas sin cobertor, gasas y algodones con sangre y secreciones corporales. Esto los sitúa dentro del personal con riesgo para contacto con material punzocortante potencialmente contaminado, al igual que al personal de mantenimiento, que manipula desechos sólidos hospitalarios.

Si bien únicamente el 3.9\% indicó desconocer las normas hospitalarias de bioseguridad, de los 76 TS que realizan procedimientos de riesgo, el $22.4 \%$ no las cumple, a pesar de que la evidencia disponible indica que el seguimiento de normas de bioseguridad disminuye el riesgo de exposición laboral al VHB (Franciscus et al., 2008; Heymann, 2011; Mendoza \& Samalvides, 2005). La estrategia más efectiva de prevención contra la infección por VHB es la vacunación y se recomienda 
que sea administrada a todos los TS (Omer, Salmon, Orenstein, deHart, \& Halsey, 2009; WHO, 2016a). Sin embargo, el $48 \%$ de cobertura de vacunación en este estudio es bajo en comparación con la cobertura estimada en TS de EUA en 2003, que fue de $75 \%$ y de hasta $81 \%$ en personal médico y de enfermería (Simard et al., 2007), con el $93 \%$ en un hospital de París (Loulergue et al., 2009) y con el $72.5 \%$ reportado en Sudáfrica (Burnett et al., 2011). La cobertura de vacunación con tres dosis en el HNPB es baja aun si se compara con países de bajo a mediano ingreso, como Nigeria, que ha alcanzado el 65\% de vacunación de los TS (Adekanle, Ndububa, Olowookere, Ijarotimi, \& Ijadunola, 2015). Una posible explicación de la baja cobertura es que antes de 2011 no existía en el país un programa nacional de vacunación dirigido a los TS. En el HNPB, la vacunación contra el VHB, se ha realizado en "jornadas" ocasionales, apoyadas por casas comerciales o por donaciones. En ocasiones, cada dosis es pagada por el propio trabajador. Es necesario fortalecer la educación sobre el autocuidado de la salud de los TS (Consejo Nacional de Salud y Seguridad Ocupacional, 2007a, 2007b) y sobre la responsabilidad del empleador y del Estado.

El porcentaje de vacunación del $48 \%$ de los TS fue cercano al $43 \%$ de seroprevalencia de anticuerpos anti-HBs; sin embargo, esta última incluye vacunados y no vacunados contra el VHB. Esto sugiere que en algunos TS los anticuerpos no fueron generados por vacunación, sino por otro tipo de contacto con el VBH. Además, no todos los vacunados generaron anticuerpos, dado que sólo el $83 \%$ de los vacunados con tres dosis tuvo una prueba anti-HBs reactiva. Aunque se describe que un 5\% de la población no responde a las tres primeras dosis, este hallazgo puede tener otras explicaciones. En primer lugar, es posible que la vacunación y la logística que implica mantener la cadena de frío, la forma de administración y la cantidad e intervalo de las dosis, no haya sido efectiva para generar inmunidad o, por lo menos, anticuerpos anti-HBs detectables. En segundo lugar, y más probablemente, la posibilidad de confusión en cuanto al tiempo y número de dosis que habían recibido los TS. En tercer lugar, la poco probable posibilidad de falsos negativos. La prueba Monolisa ${ }^{\circledR}$ Anti-HBs PLUS tiene una sensibilidad de 99.2\% (IC $95 \%, 98.1-99.7$ ) y una especificidad de $99.4 \%$ (IC $95 \%$, 98.8 s 99.8); el límite inferior de confianza de la especificidad indica que el porcentaje de falsos negativos puede ser de hasta un máximo de $1.2 \%$, lo que sugiere una baja probabilidad de que esto explique la falta de reactividad.
La vacunación incompleta se acompañó de una menor reactividad a la prueba anti-HBs, tal como está descrito en la literatura. De 29 TS que reportaron haber recibido tres dosis de vacuna, $23(79 \%)$ fueron reactivos y seis $(21 \%)$, no reactivos. En comparación, de 12 vacunados con dos dosis, siete (58\%) tuvieron una prueba reactiva y cinco ( $42 \%$ ) una prueba no reactiva, mientras que de siete vacunados con una única dosis, hubo dos $(29 \%)$ con prueba reactiva y cinco $(71 \%)$ con prueba no reactiva. De ahí la importancia de medir concentración de anticuerpos para determinar si el TS tiene niveles protectores contra VHB (Chien et al., 2014).

Por el contrario, de 48 que reportaron no estar vacunados, $11(23 \%)$ tuvieron una prueba anti-HBs reactiva. Una mala clasificación de la exposición (haber reportado que no, cuando sí recibieron la vacuna) pudo introducir un error de información, específicamente de memoria, que no pudo ser eliminado en este estudio debido a la falta de una fuente más confiable de datos, por ejemplo, registros de vacunación, lo que limitó la interpretación de estas 11 pruebas reactivas. En un paciente no vacunado, este hallazgo tiene tres explicaciones biológicamente plausibles: que haya tenido una infección pasada por VHB, que se trate de un resultado falso positivo o que no recuerde que fue vacunado (Shepard, Simard, Finelli, Fiore, \& Bell, 2006). Para confirmar una infección pasada se hace una prueba de anticuerpos contra el antígeno del core, anti-HBc, mientras que para descartar un falso positivo habría que repetir la prueba. Algo similar sucede con los 15 que indicaron no saber si están vacunados, de los cuales cuatro $(27 \%)$ fueron reactivos. Tanto en la totalidad de la muestra de 121 sujetos, como en el subgrupo de 58 sujetos con mayor riesgo de contagio, la prevalencia de anti-HBs aumentó con el número de dosis, desde los no vacunados $(23 \%$ y $26 \%$, respectivamente) hasta los vacunados con tres dosis ( $79 \%$ y $86 \%$, respectivamente).

De 69 participantes con prueba anti-HBs no reactiva, $21(30 \%)$ indicaron haber sido vacunados contra el VHB. De 10 que respondieron haber sido vacunados, pero no saben cuántas dosis, cinco $(50 \%)$ tuvieron una prueba reactiva. Finalmente, de 15 que no saben si fueron vacunados, cuatro $(27 \%)$ dieron positivo para anti-HBs. Estos resultados resaltan la necesidad de fortalecer los programas educativos sobre SSO, así como de crear el registro de vacunación de TS. Ante resultados discordantes, se recomienda repetir la prueba y medir marcadores serológicos adicionales para hacer el diagnóstico (Dufour \& Lott, 2005). 
Una asociación entre incidente punzocortante y el tipo de exposición ocupacional, como contacto directo con pacientes manipulación de material biológico y punzocortante (Tabla 2), ha sido descrita en otros estudios, en los que paramédicos, enfermeras universitarias, auxiliares y médicos tuvieron la mayor cantidad de incidentes y, a la vez, más contacto con pacientes y con material biológico y punzocortante (Martínez, Alarcón, Lioce, Tennasse, \& Wuilburn, 2008; C. Mendoza et al., 2001; Organización Panamericana de la Salud [OPS], 2002). Sin embargo, las comparaciones entre los resultados de diferentes estudios se dificultan debido a la heterogeneidad de las categorías y mediciones usadas.

Se encontró asociación significativa entre el riesgo de lesión ocupacional por objetos punzocortantes y la profesión médica, así como por el cargo de enfermería, ambos considerados grupos de alto riesgo dentro de los TS en otros estudios (Wilburn \& Eijkemans, 2004).

Se preguntó sobre la afiliación al IGSS porque, para cuando se realizó el estudio, la vacuna contra el VHB no estaba disponible en los servicios públicos de salud en todo momento; la información fue útil para recomendar a los participantes que buscaran la administración de la vacuna en el IGSS. Del total de participantes, 103 (85\%) cotizaban al IGSS, el 57\% de ellos tuvo resultado negativo para anti-HBs, de los que 24 reportaron contacto con pacientes o con desechos biológicos.

Las prácticas de bioseguridad e inmunización encontradas entre los TS del hospital fueron deficientes. El porcentaje de TS sin anticuerpos es importante, por lo que se recomienda vacunar a los TS negativos y discutir las posibles medidas para asegurar que completen el esquema. Se recomienda fortalecer las competencias sobre bioseguridad y el cumplimiento de medidas preventivas en el hospital, así como institucionalizar el acceso a la vacunación contra VHB para todos los TS. Se recomienda hacer estudios complementarios de anti-HBc a los TS positivos sin historia de vacunación, así como concentraciones de anticuerpos anti-HBs antes de ingresar a trabajar al hospital.

Limitaciones del estudio. Aunque la composición de la muestra de estudio es similar a la de la población trabajadora del hospital reportada previamente (Velásquez \& Partanen, 2007), la participación de sujetos voluntarios es una fuente potencial de sesgo de selección, ya que estos tienden a mostrar conductas de autocuidado diferentes de los no voluntarios (Gordis, 2014). En las asociaciones halladas entre diversos fac- tores y accidente ocupacional punzocortante, hay que tener siempre en cuenta la posibilidad del sesgo de memoria, ya que la experiencia misma del accidente puede influir en un mejor recuerdo de la exposición (Gerhard, 2008). No obstante, este estudio auxiliar es una estrategia metodológica válida en la que "el investigador añade una o varias determinaciones a un estudio existente para responder a una pregunta diferente" (Randy $\&$ Hearst, 2008). El diseño transversal no permite establecer una secuencia temporal entre exposición y desenlace, por ello, las asociaciones encontradas en este estudio son estadísticas y no causales. La detección de anticuerpos anti-HBs se hizo de forma cualitativa (reactivo/no reactivo), lo que no nos permitió medir la concentración de anticuerpos para determinar si existen niveles protectores.

\section{Agradecimientos}

Los autores agradecen a la Dirección, miembros del Comité de Infecciones Nosocomiales, Dirección y personal del laboratorio y a la Dirección y personal del Departamento de Nutrición del HNPB. Asimismo, a la Dirección y al personal del Laboratorio Multidisciplinario de la Facultad de Ciencias Médicas de la Usac, por sus valiosos aportes técnicos.

\section{Referencias}

Adekanle, O., Ndububa, D., Olowookere, S., Ijarotimi, O., \& Ijadunola, K. (2015). Knowledge of hepatitis $B$ virus infection, immunization with hepatitis $B$ vaccine, risk perception, and challenges to control hepatitis among hospital workers in a Nigerian tertiary hospital. Hepatitis Research and Treatment, 2015, 1-6. doi:10.1155/2015/439867

Ben-Ari, Z., Weitzman, E., \& Safran, M. (2015). Oncogenic viruses and hepatocellular carcinoma. Clinics in Liver Disease, 19(2), 341-360. doi:10.1016/j.cld.2015.01.006

Burnett, R. J., François, G., Mphahlele, M. J., Mureithi, J. G., Africa, P. N., Satekge, M. M., ... van Sprundel, M. (2011). Hepatitis B vaccination coverage in healthcare workers in Gauteng Province, South Africa. Vaccine, 29(25), 42934297. doi:10.1016/j.vaccine.2011.03.001 
Chacaltana, A., \& Espinoza, J. (2008). Seroprevalencia de la infección y factores de riesgo para hepatitis B y $\mathrm{C}$ en personal militar sano. Revista de Gastroenterología del Perú, 28, 217-225.

Chien, Y. C., Jan, C. F., Chiang, C. J., Kuo, H. S., You, S. L., \& Chen, C. J. (2014). Incomplete hepatitis $\mathrm{B}$ immunization, maternal carrier status, and increased risk of liver diseases: A 20-year cohort study of 3.8 million vaccinees. Hepatology, 60(1), 125-132. doi:10.1002/hep. 27048

Consejo Nacional de Salud y Seguridad Ocupacional. (2007a). Encuesta nacional sobre condiciones de trabajo, salud y seguridad ocupacional. Guatemala: Autor.

Consejo Nacional de Salud y Seguridad Ocupacional. (2007b). Perfil diagnóstico nacional sobre condiciones de trabajo, salud y seguridad ocupacional. Guatemala: Autor.

Cubides, I., \& Suárez, C. Y. (2009). Epidemiología e historia natural de la hepatitis B. Revista Colombiana de Gastroenterología, 24, 4s-12s.

Dufour, D. R., \& Lott, J. A. (2005). Guías del laboratorio para screening, diagnóstico y monitoreo de la lesión hepática. Acta Bioquímica Clínica Latinoamericana, 39(3), 359-376.

El-Serag, H. B. (2011). Hepatocellular carcinoma. New England Journal of Medicine, 365(12), 11181127. doi:10.1007/s10354-014-0296-7

Franciscus, A., Highleyman, L., \& Kukka, C. (2008). Guía para comprender la Hepatitis B (Versión 4.1). San Francisco, California: Hepatitis C Support Project.

Gerhard, T. (2008). Bias: Considerations for research practice. American Journal of Health-System Pharmacy, 65(22), 2159-2168. doi:10.2146/ ajhp070369

Gordis, L. (2014). Epidemiology (5th ed.). Philadelphia: Elsevier Saunders.

Halabe, J., \& Angulo, F. (2001). Hepatitis viral. Medicina Interna de México, 43(3), 90-100. doi:10.4067/S0034-98872009000100020

Heymann, D. (2011). El control de las enfermedades transmisibles (19th ed.). Washington, DC.: OPS/ OMS .
Lewis, J. D., Enfield, K. B., \& Sifri, C. D. (2015). Hepatitis B in healthcare workers: Transmission events and guidance for management. World Journal of Hepatology, 7(3), 488-497. doi:10.4254/wjh.v7.i3.488

Lok, A. S. F., \& McMahon, B. J. (2009). Chronic hepatitis B: Update 2009. Hepatology, 50(3), 661-662. doi:10.1002/hep. 23190

Loulergue, P., Moulin, F., Vidal-Trecan, G., Absi, Z., Demontpion, C., Menager, C., ... Launay, O. (2009). Knowledge, attitudes and vaccination coverage of healthcare workers regarding occupational vaccinations. Vaccine, 27(31), 42404243. doi:10.1016/j.vaccine.2009.03.039

Martínez, M., Alarcón, W., Lioce, M., Tennasse, M., \& Wuilburn, S. (2008). Prevención de accidentes laborales con objetos punzocortantes y exposición ocupacional a agentes patógenos de la sangre en el personal de salud. Salud de Los Trabajadores, 16(1), 53-62.

McMahon, B. J. (2005). Epidemiology and natural history of hepatitis B. Seminars in Liver Disease, 25(Suppl) 1, 3-8. doi:10.1055/s-2005-915644

Mendoza, A., \& Samalvides, F. (2005). Transmisión de los virus de la inmunodeficiencia adquirida, hepatitis B y hepatitis $\mathrm{C}$ por exposiciones laborales en trabajadores de salud: Aspectos de profilaxis pre y post exposición. Revista Médica Herediana, 16(4), 276-284.

Mendoza, C., Barrientos, C., Vasquez, R., \& Panizza, V. (2001). Exposición laboral a sangre y fluidos corporales: Experiencia en un hospital pediátrico. Revista Chilena de Infectología, 18(1), 28-34. doi:10.4067/S0716-10182001000100004

Morelos, R., Ramírez, M., Sánchez, G., Chavarín, C., \& Meléndez-Herrada, E. (2014). El trabajador de la salud y el riesgo de enfermedades infecciosas adquiridas. Revista de La Facultad de Medicina de La UNAM, 57(4), 34-42.

Naghavi, M., Wang, H., Lozano, R., Davis, A., Liang, X., Zhou, M., ... Temesgen, A. M. (2015). Global, regional, and national age-sex specific all-cause and cause-specific mortality for 240 causes of death, 1990-2013: A systematic analysis 
for the Global Burden of Disease Study 2013. Lancet, 385(9963), 117-171. doi:10.1016/S01406736(14)61682-2

Omer, S. B., Salmon, D. A., Orenstein, W. A., deHart, M. P., \& Halsey, N. (2009). Vaccine refusal, mandatory immunization, and the risks of vaccine-preventable diseases. New England Journal of Medicine, 360(19), 1981-1988. doi:10.1056/NEJMsa0806477

Organización Mundial de la Salud. (12 de noviembre de 2009). Hepatitis virales. Informe de la Secretaría. Consejo Ejecutivo 126. Reunión, Punto 4.12 del orden provisional (EB126/15). Recuperado de http://apps.who.int/gb/ebwha/pdf_files/EB126/ B126_15-sp.pdf

Organización Panamericana de la Salud. (2002). Exposición laboral a los virus de la hepatitis $\mathrm{B}$ y $\mathrm{C}$ $\mathrm{y}$ al virus de la inmunodeficiencia humana. Revista Panamericana de Salud Pública, 11(2), 132-141. doi:10.1590/S1020-49892002000200016

Ott, J. J., Stevens, G. A., Groeger, J., \& Wiersma, S. T. (2012). Global epidemiology of hepatitis B virus infection: New estimates of age-specific $\mathrm{HBsAg}$ seroprevalence and endemicity. Vaccine, 30(12), 2212-2219. doi:10.1016/j.vaccine.2011.12.116

Randy, D., \& Hearst, N. (2008). Utilización de las bases de datos existentes. En S. B. Hulley, S. R. Cummings, W. S. Browner, D. G. Grady, \& T. B. Newman (Eds.), Diseño de investigaciones clínicas (3rd ed., pp. 233-249). Barcelona: Wolters Kluwer Lippincott Williams \& Wilkins.

Schweitzer, A., Horn, J., Mikolajczyk, R. T., Krause, G., \& Ott, J. J. (2015). Estimations of worldwide prevalence of chronic hepatitis B virus infection: A systematic review of data published between 1965 and 2013. Lancet, 386(10003), 1546-1555. doi:10.1016/S0140-6736(15)61412-X

Shepard, C. W., Simard, E. P., Finelli, L., Fiore, A. E., $\&$ Bell, B. P. (2006). Hepatitis B virus infection: Epidemiology and vaccination. Epidemiologic Reviews, 28(1), 112-125. doi:10.1093/epirev/ mxj009

Simard, E., Miller, J., George, P., Wasley, A., Alter, M., Bell, B., \& Finelli, L. (2007). Hepatitis B vaccination coverage levels among healthcare workers in the United States, 2002-2003. Infection Control and Hospital Epidemiology, 28(7), 783790. doi:10.1086/518730

Suárez, M., Mursuli, I., Pérez, Y., \& Sánchez, A. (2005). Factores de riesgo en portadores de hepatitis B. Revista Mexicana de Patología Clínica, 52(1), 5358.

Tanaka, J. (2000). Hepatitis B epidemiology in Latin America. Vaccine, 18(Suppl. 1), 19-21. doi:10.1016/S0264-410X(99)00455-7

Trépo, C., Chan, H. L. Y., \& Lok, A. (2014). Hepatitis B virus infection. Lancet, 384(9959), 2053-2063. doi:10.1016/S0140-6736(14)60220-8

Velásquez, M., \& Partanen, T. (2007). Promoción de la salud del personal que labora en el Hospital "Pedro de Bethancourt", Antigua Guatemala: Fase diagnóstica, (Serie Salud y Trabajo, № 6). Heredia, Costa Rica. Recuperado de http://www. repositorio.una.ac.cr/handle/11056/8594

Weinbaum, C. M., Mast, E. E., \& Ward, J. W. (2009). Recommendations for identification and public health management of persons with chronic hepatitis B virus infection. Hepatology, 49 (Suppl. 5), 35-44. doi:10.1002/hep.22882

Wilburn, S., \& Eijkemans, G. (2004). Preventing needlestick injuries among healthcare workers. International Journal of Occupational and Environmental Health, 10(4), 451-456. doi:10.1179/oeh.2004.10.4.451

World Health Organization. (2016a). Global health sector strategy on viral hepatitis 2016-2021: Towards ending viral hepatitis. Geneva, Switzerland: Global Hepatitis Programme Department of HIV/AIDS

World Health Organization. (2016b). Hepatits B. Recuperado de http://www.who.int/mediacentre/ factsheets/fs204/en/

Zumaeta, E., González, A., Ferrandiz, A., Villanueva, A., Soto, V., Almeida, R., ... Anton, M. (2001). Pronóstico de duración de títulos protectores anti-HBs ag en trabajadores de la salud del perú luego de 6 años de vacunados. Revista de Gastroenterología del Perú, 21, 276-281. 